\section{S112 ESTIMATING PARTICIPATION RATES OF COPD PATIENTS IN PULMONARY REHABILITATION AND SELF-MANAGEMENT PROGRAMMES: THE IMPORTANCE OF DEFINING PARTICIPATION}

doi:10.1136/thoraxjnl-2012-202678.117

R Sohanpal, RL Hooper, R Hames, S Priebe, SJC Taylor. Barts and The London School of Medicine and Dentistry, Queen Mary University of London, London, UK

Introduction and Objectives Delivery of pulmonary rehabilitation (PR) and self-management (SM) support programmes to patients living with chronic obstructive pulmonary disease (COPD) is emphasised in national guidelines, but these interventions are not always routinely available. One potential explanation for the lack of implementation could be the poor patient participation and retention reported in the literature. We conducted a systematic review to determine a true estimate of participation and dropout rates.

Methods Studies were identified from eight electronic databases including MEDLINE. Controlled clinical trials of structured SM, PR and health education (HE) programmes for COPD were included. Data extraction included 'participant flow' data using the Consolidated Standards of Reporting Trials (CONSORT) statement and its extension to pragmatic trials. Patient 'participation rates' (study participation rate (SPR), study dropout rate (SDR) and intervention dropout rate (IDR)) were calculated using prior definitions consistent with CONSORT Random effects logistic regression analysis was conducted to examine effects of four key study characteristics on participation rates.

Results 56 quantitative studies (51 randomised controlled trials, three quasi-experimental and two before-after studies) evaluated PR $(n=31), S M(n=21)$ and HE $(n=4)$. Reports of participant flow were generally incomplete; 'numbers of potential participants identified' were only available for $16 \%$, and 'numbers assessed for eligibility' for only $39 \%$ of studies. Although 'numbers eligible' were better reported (77\%), we were unable to calculate SPR for $23 \%$ of studies.

Overall we found 'participation rates' for studies $(n=43)$ were higher than previous reports; only $19 \%$ of studies had less than $50 \%$ SPR and just over one third (34\%) had a SPR of 100\%; SDR and IDR were less than or equal to $30 \%$ for around $93 \%$ of studies. There was no evidence of effect of study characteristics on participation rates. Conclusion Unlike previous reports, we found high participation and low dropout rates in studies of PR or SM support for COPD Previous studies adopted different participation definitions; some reported proportions without stating definitions clearly, obscuring whether proportions referred to the study or the intervention. Clear, uniform definitions of patient participation in studies are needed to better inform the wider implementation of effective interventions.

\section{S113 LONG TERM EXERCISE (LTE) FOR COPD PATIENTS POST-PULMONARY REHABILITATION (PR) PROLONGS THE DURATION OF BENEFITS DERIVED FROM PR}

doi:10.1136/thoraxjnl-2012-202678.118

${ }^{1} \mathrm{SE}$ Roberts, ${ }^{2} \mathrm{~S}$ Rogers, ${ }^{2} \mathrm{~A}$ Segal, ${ }^{3} \mathrm{~S}$ Purcell, ${ }^{3} \mathrm{H}$ Broomfield, ${ }^{3} \mathrm{G}$ Fabris, ${ }^{4} \mathrm{M}$ CalongeContreras, ${ }^{4} \mathrm{~J}$ Billet, ${ }^{3} \mathrm{~L}$ Restrick, ${ }^{3} \mathrm{M}$ Stern. ${ }^{1}$ Dept. Physiotherapy, Whittington Health, London, United Kingdom; 'Independent Exercise Instructors, London, United Kingdom; ${ }^{3}$ Department Respiratory Medicine, Whittington Health, London UK, London, United Kingdom; ' ${ }^{D}$ Dept. Public Health, London Borough of Islington/NHS North Central, London, United Kingdom

Introduction PR is associated with functional, emotional and resource benefits for breathless COPD patients which decline over 12-18 months. Patients who complete PR express the desire to continue exercising regularly with other breathless patients but there is currently no evidence for efficacy. This study evaluated regular community-based LTEfor these patients.
Methods Patients completing PR were recruited to once weekly LTE held in accessible venues by 2 exercise instructors (Loughborough trained for exercising patients with chronic respiratory disease). Baseline demographics and disease severity were collected and outcomes: 6 minute walk test (6MWT), Hospital Anxiety \& Depression (HAD) score, COPD Assessment Test (CAT), Chronic Respiratory Questionnaire (CRQ) and patient satisfaction measured at baseline, 6 and 12 months. Patients who accepted referral for LTE but never attended or dropped-out were recalled for outcomes at 12 months. Hospital admissions were audited for 12 months after PR-completion.

Results Between June-2010 and January-2012 75 patients mean(SD) age 69.3(9.7)yrs, FEV 1.26(0.54)L, MRC 3.16(0.81) 63\% female, $19.2 \%$ current smokers and 3 on LTOT accepted referral to LTE. 35\% (26/75) never attended and 27\%(20/75) dropped out after starting; 39\% (29/75) continued to exercise for at least 6 months and $25 \%(19 / 75)$ exercised to 1 yr. For patients who exercised for 12 months there was no significant decline in exercise capacity (6MWT), a significant improvement in CAT over $6 / 12(p=0.002)$ maintained to $12 / 12(p=0.02)$ and no increase in anxiety levels, which remained below clinical relevance for the 12 months post PR. In comparison, patients who did not continue LTE had a significant $(p=0.001)$ decline in 6MWT, no change in CAT score and a significant $(p=0.04)$ increase in anxiety to a clinically important range (table 1). Self reported hospital admissions in the year following PR were higher for patients who did not exercise (mean 0.61 (SD 1.47)) compared to those who did, 0.16 (0.50).

Conclusions This pilot demonstrates that community-based LTE with trained instructors is safe and realistic for breathless patients after completing PR and, for the first time, demonstrates significant prolongation of functional and emotional benefits. This offers acheaper, more durable alternative to repeating PR.

\section{Inflammatory cell phenotype and activation in asthma}

\section{S114 ADAPTIVE AND INNATE-LIKE T CELL PHENOTYPES IN ASTHMA IN RELATIONSHIP TO COMPARTMENT AND SEVERITY}

doi:10.1136/thoraxjnl-2012-202678.119

'TSC Hinks, 'KJ Staples, 'C Smith, 'J Ward, 'S Mansour, P Monk', 'PH Howarth, ${ }^{3} \mathrm{HW}$ Virgin, 'S Gadola, $1 \mathrm{R}$ Djukanovic. 'Academic Unit of Clinical and Experimental Sciences, Faculty of Medicine, University of Southampton School of Medicine, Southampton, UK; ${ }^{2}$ Synairgen Research Ltd, Mail Point 810, Southampton General Hospital, Southampton, UK; ${ }^{3}$ Department of Pathology and Immunology, Washington University School of Medicine, St Louis, USA

Introduction and Objectives Asthma is a heterogeneous disease affecting 150-300 million people. Underlying mechanisms remain unclear. $T_{H} 17$ cells expressing interleukin-17 are widely hypothesised to play a role, particularly in severe neutrophilic asthma. Mucosal associated invariant T (MAIT) cells are novel innate-like T-cells of unknown function which express CD161 and an invariant TCR $\alpha$ chain (V $\alpha 7.2-J \alpha 33)$ and recognise the highly conserved restriction molecule MR1.

We undertook to analyse IL-17 and key T-cell subsets, namely $\mathrm{T}_{\mathrm{H}} 17, \mathrm{~T}_{\mathrm{H}} 1, \mathrm{~T}_{\mathrm{H}} 2, \mathrm{~T}_{\mathrm{REG}}$ and MAIT cells in relation to asthma severity and virus-induced exacerbations.

Methods Cross sectional study: 76 subjects underwent detailed phenotyping, sputum induction, phlebotomy, and bronchoscopy. Samples were analysed by 9-colour flow-cytometry, RT-PCR, multiplex ELISA, microarray, and deep sequencing of the airway microbiome. Longitudinal study: 35 frequently exacerbating asthmatics: followed at 7 time-points during a naturally occurring cold. 